\title{
Tracking the spread of a passive tracer through Southern Ocean water masses
}

\author{
Jan D. Zika ${ }^{1}$, Jean-Baptiste Sallée ${ }^{2}$, Andrew J. S. Meijers ${ }^{3}$, Alberto C. Naveira-Garabato ${ }^{4}$, Andrew J. Watson ${ }^{5}$, \\ Marie-Jose Messias ${ }^{5}$, and Brian A. King ${ }^{6}$ \\ ${ }^{1}$ School of Mathematics and Statistics, University of New South Wales, Sydney, Australia \\ ${ }^{2}$ CNRS, LOCEAN Laboratory, Sorbonne Université, Paris, France \\ ${ }^{3}$ British Antarctic Survey, Cambridge, UK \\ ${ }^{4}$ National Oceanography Centre, University of Southampton, Southampton, UK \\ ${ }^{5}$ College of Life and Environmental Sciences, University of Exeter, Exeter, UK \\ ${ }^{6}$ National Oceanography Centre, Southampton, UK
}

Correspondence: Jan D. Zika (j.zika@unsw.edu.au)

Received: 27 August 2019 - Discussion started: 30 August 2019

Revised: 16 January 2020 - Accepted: 16 January 2020 - Published: 12 March 2020

\begin{abstract}
A dynamically passive inert tracer was released in the interior South Pacific Ocean at latitudes of the Antarctic Circumpolar Current. Observational cross sections of the tracer were taken over 4 consecutive years as it drifted through Drake Passage and into the Atlantic Ocean. The tracer was released within a region of high salinity relative to surrounding waters at the same density. In the absence of irreversible mixing a tracer remains at constant salinity and temperature on an isopycnal surface. To investigate the process of irreversible mixing we analysed the tracer in potential density-versus-salinity-anomaly coordinates. Observations of high tracer concentration tended to be collocated with isopycnal salinity anomalies. With time, an initially narrow peak in tracer concentration as a function of salinity at constant density broadened with the tracer being found at ever fresher salinities, consistent with diffusion-like behaviour in that coordinate system. The second moment of the tracer as a function of salinity suggested an initial period of slow spreading for approximately 2 years in the Pacific, followed by more rapid spreading as the tracer entered Drake Passage and the Scotia Sea. Analysis of isopycnal salinity gradients based on the Argo programme suggests that part of this apparent change can be explained by changes in background salinity gradients while part may be explained by the evolution of the tracer patch from a slowly growing phase where the tracer forms filaments to a more rapid phase where the tracer mixes at $240-550 \mathrm{~m}^{2} \mathrm{~s}^{-1}$.
\end{abstract}

\section{Introduction}

Isopycnal mixing is a key controller of the transport of carbon (Sallée et al., 2012; Gnanadesikan et al., 2015) and heat (Gregory, 2000) into the deep sea, and of the stability of the long-term climate system (Sijp et al., 2006). The influence of isopycnal mixing is nowhere more prevalent than in the Southern Ocean, where steeply sloping isopycnals form a connection between the atmosphere and the deep ocean (Marshall and Speer, 2012). Strong gradients of temperature and salinity along isopycnals imply a necessary balance between advection of relatively warm saline Circumpolar Deep Water and its freshening by isopycnal and diapycnal mixing (Zika et al., 2009b; Naveira Garabato et al., 2016). Here we explore how a passive tracer released as part of the Diapycnal and Isopycnal Mixing Experiment in the Southern Ocean (DIMES; http://dimes.ucsd.edu, last access: 9 March 2020) project mixes through this background salinity and temperature gradient.

Previous tracer release experiments have found that tracers closely followed the release isopycnal surface, spreading slowly across isopycnal surfaces (i.e. across vertically stacked density layers; Ledwell et al., 1993, 2011; Morris et al., 2001; Watson et al., 2013). Tracer profiles in these studies were typically averaged over sections as a function of density and then transformed into depth coordinates through a reference density-vs.-depth profile. The motivation for averaging at constant density is that the tracer can 
move to different depth levels through adiabatic reversible motions. Moving to different density values in the ocean interior (i.e. away from sources and sinks of heat and salt) requires irreversible mixing, which either directly mixes the tracer across isopycnals or causes diapycnal advection (e.g. Toole and McDougall, 2001).

Just as vertical displacements of the tracer can occur without diapycnal mixing, lateral spreading of the tracer can occur without any irreversible mixing. The tracer can be spread out or contract geographically due to diverging or converging ocean currents, respectively. If such dynamics are reversible, as would be expected from laminar changes in the Antarctic Circumpolar Current (ACC) along its path or broad wavelike fluctuations in frontal positions, the concentration of the tracer at constant density and salinity should not change. The exception to this is when the flow "stirs" the tracer.

Typically a distinction is made between "stirring", which is associated with mesoscale and sub-mesoscale motions creating long filaments and sharp gradients, and "mixing", which relates to the resultant irreversible mixing once filaments have become sufficiently sharp that small-scale turbulent mixing is sufficient to mix water masses irreversibly (Smith and Ferrari, 2009). The two are linked since stirring enables mixing. Garrett (1983) showed that with background small-scale mixing held constant, the rate of growth of the area of an ensemble of tracer patches (a measure of the rate of stirring) approaches the rate of growth of the area of a single tracer patch (a measure of irreversible mixing) after some initial lag time of the order of years. This behaviour was consistent with both tracer and Lagrangian float observations during the North Atlantic Tracer Release Experiment (Ledwell et al., 1998; Sundermeyer and Price, 1998).

Because of the density of observations we will consider, we cannot estimate the area of our tracer patch accurately, so we will project the data onto a coordinate system which preserves adiabatic horizontal or isopycnal motions just as isopycnal coordinates preserve adiabatic vertical motions.

Various cross-stream coordinates have been proposed, such as sea surface height (Sokolov and Rintoul, 2009; Meijers et al., 2011), dynamic height (Naveira Garabato et al., 2011) and density on pressure surfaces (Zika et al., 2013). However, these are not necessarily conserved following the tracer path and implicitly assume that the flow is "equivalentbarotropic" throughout the entire water column (Killworth, 1992). Any subtle spiralling of the velocity vector with depth can accumulate to create a mismatch between the flow direction on a particular isopycnal and the tangent lines of sea surface height (SSH) or dynamic height contours at the surface.

It has long been understood that, in the absence of irreversible mixing, a water parcel in the ocean interior will remain at constant salinity and temperature (Iselin, 1939). Since the mixing of water parcels with the same density is thought to dominate over mixing at different densities, a variable known as "spice" is often used to track along-isopycnal motion. Under the assumption of a linear equation of state, spice describes the density-compensated variations in temperature and salinity along a constant density surface and is orthogonal to density in the temperature-salinity plane (Veronis, 1972). The spice variable has been exploited by a number of authors (e.g. Rudnick and Martin, 2002).

Here we simply use salinity at constant density as our coordinate which is equivalent locally to a typical definition of spice. Since density is locally a function of conservative temperature and salinity, a line of constant salinity on an isopycnal is also a line of constant temperature (Zika et al., 2009a). So our analysis would be the same whether we consider temperature or salinity as our horizontal coordinate. In either case, there is no way a tracer in the ocean interior, which is released on such a contour, can move away from that contour without irreversible mixing.

We have analysed tracer data collected during DIMES using an isopycnal salinity coordinate with the aim of helping to understand isopycnal dispersion and complementing previous studies that have used more conventional approaches to estimate isopycnal mixing rates. Using these data Tulloch et al. (2014) estimated the isopycnal diffusion coefficient to be $710 \pm 260 \mathrm{~m}^{2} \mathrm{~s}^{-1}$ along the ACC in the South Pacific Ocean near the release depth of $1500 \mathrm{~m}$. Those authors applied the ensemble tracer patch area approach (Garrett, 1983). In a parallel study, LaCasce et al. (2014) analysed data from subsurface Lagrangian drifters released during the DIMES campaign. They estimated the isopycnal diffusivity to be $800 \pm 200 \mathrm{~m}^{2} \mathrm{~s}^{-1}$ using the Lagrangian dispersion method (Garrett, 1983).

We show, in Sect. 2, that the tracer released in the interior South Pacific spreads geographically in an inhomogeneous way as it follows the ACC and is stirred by eddies. In Sect. 3, we show that some of this inhomogeneity is reduced when the tracer is projected into salinity coordinates. In Sect. 4, we project the spreading of the tracer in salinity coordinates into equivalent geographical coordinates. We then attempt to reconcile the growth of its second moments in equivalent geographical coordinates in terms of diffusion coefficients in Sect. 5, and we discuss these results in terms of existing theories in Sect. 6. Concluding remarks are given in Sect. 7.

\section{Tracer data}

In February $2009,76 \mathrm{~kg}$ of the inert tracer trifluoromethyl sulfur pentafluoride $\left(\mathrm{CF}_{3} \mathrm{SF}_{5}\right)$ was released at the potential density (referenced to $1000 \mathrm{~m}$ ) of $32.325 \mathrm{~kg} \mathrm{~m}^{-3}$ (neutral density $\approx 27.9 \mathrm{~kg} \mathrm{~m}^{-3}$; depth $\approx 1500 \mathrm{~m}$ ) near $58^{\circ} \mathrm{S}, 253^{\circ} \mathrm{E}$. The tracer was chosen as it has a very low background atmospheric concentration and nearly negligible interior ocean concentration, is non-toxic and is chemically inert in the environment, and is detectable at extreme dilution (Ho et al., 2008). The tracer was released in a cross pattern approximately $20 \mathrm{~km}$ wide, between the Subantarctic and Polar 
fronts and at the density of Upper Circumpolar Deep Water. The initial tracer distribution, determined by sampling within 2 weeks of the release, was found to be centred about $4 \mathrm{~m}$ below the target density surface. The rms spread of the tracer in density was documented as approximately $0.0015 \mathrm{~kg} \mathrm{~m}^{-3}$, corresponding to a vertical rms spread of $5.5 \mathrm{~m}$. For details of the release method and measurement technique, see Ledwell et al. (2011).

Several subsequent surveys were undertaken: in February 2010 in the region 57 to $62^{\circ} \mathrm{S}$ and 255 to $275^{\circ} \mathrm{E}$ (Fig. 1a); in December 2010 (Fig. 1b), April 2011 (Fig. 1c) and August 2011 (Fig. 1d) in the regions west of and at Drake Passage; and along the western and northern margins of the Scotia Sea in 2012 (February-March, Fig. 1e) and 2013 (MarchApril, Fig. 1f) to measure the change in distribution of the tracer with time. Tracer sampling and shipboard chemical analysis are described in Ledwell et al. (2011) and in Watson et al. (2013).

We have analysed 11 sections taken at 5 locations, here named as follows: South Pacific (actually a regional survey), south-east Pacific (along $282^{\circ} \mathrm{E}$ ), Drake Entry (near $293^{\circ} \mathrm{E}$ ), Drake exit (along the section commonly termed SR1b near $303^{\circ} \mathrm{E}$ ), and North Scotia (along the North Scotia Ridge). These locations are indicated in Fig. 1.

Year on year, the tracer was found increasingly toward the east and in lower concentrations (Fig. 2; Watson et al., 2013). The tracer closely followed the release isopycnal surface, spreading slowly across isopycnal surfaces (i.e. across vertically stacked density layers). Tracer profiles averaged over sections as a function of density and then transformed into depth coordinates through a reference density-depth profile were approximately Gaussian. The spread of these Gaussian profiles with time and/or with distance to the east yielded diapycnal diffusivities of $\mathrm{O}\left(2 \times 10^{-5}\right) \mathrm{m}^{2} \mathrm{~s}^{-1}$ in the South Pacific (Ledwell et al., 2011; Watson et al., 2013) and $\mathrm{O}\left(3 \times 10^{-4}\right) \mathrm{m}^{2} \mathrm{~s}^{-1}$ in the Scotia Sea, where the ACC flows over comparatively shallow and complex topography (Watson et al., 2013).

The tracer was not always delimited at the northern and southern ends of the transects or surveys due to compromises dictated by ship time limitations. Sampling was planned with information on the locations of the Polar Front to the south and the Subantarctic Front (west of Drake Passage) or of the continental slope (east of Drake Passage) to the north. The tracer had been released midway between the two fronts, and so when compromises in sampling were needed, sampling was curtailed beyond the fronts, especially north of the Subantarctic Front in the Pacific, guided by the notion that the fronts are barriers to cross-ACC transport (e.g. Naveira Garabato et al., 2016) and also by an altimetry-based prediction of tracer patch spreading. It turned out that little tracer was detected south of the Polar Front when time allowed for sampling there. However, sections in the Pacific often found high concentrations in the northernmost (although still south of the Subantarctic Front) stations, suggesting that there was tracer to the north of the sampled area. In fact, the evidence is that not very much of the tracer spread to the north of the Subantarctic Front. The DIMES float trajectories, shown in Fig. 3 of LaCasce et al. (2014), illustrate that virtually none of the floats went far enough north to avoid transiting through Drake Passage. The ensemble of numerical simulations for realistic conditions, calibrated with the available tracer observations, shown in Fig. 1 of Tulloch et al. (2014) also suggests that relatively little tracer was missed to the north or south of the sampled regions. Hence, we argue that tracer sampling was sufficiently representative to support our main conclusions, although the shortfall of sampling adds to the uncertainty of our quantitative analysis.

The tracer was apparently distributed over a smaller meridional range at the Drake Passage exit section than in the south-east Pacific. This could be due to a decrease in meridional spread as the tracer moves downstream associated with the contraction of the ACC as it flows through Drake Passage or due to sampling issues. In either case it poses a problem to conventional analysis of cross-ACC dispersion in terms of a Fickian eddy diffusivity since the second moment in geographical space decreases with time. We shall see shortly that analysis of the along-isopycnal spreading of the tracer into waters with different salinities helps to circumvent this problem.

\section{Tracer dispersion in density-versus-salinity-anomaly coordinates}

The tracer was found to spread inhomogeneously in the horizontal. Figure 3 shows the distribution of the tracer as a function of latitude and depth (panels a-c) and latitude and density $(\mathrm{d}-\mathrm{f})$ and in temperature and salinity coordinates $(\mathrm{g}-\mathrm{i})$ for the South Pacific section in February 2010 (a, d and g), the south-east Pacific section in December 2010 (b, e and h) and the Drake exit section in March 2011 (c, f and i).

The tracer spread isopycnally and formed multiple peaks in concentration, suggesting that the patch had been teased into filaments. When the tracer distribution is projected onto temperature-versus-salinity coordinates, these individual maxima are not seen, suggesting that filaments largely preserve their temperature and salinity values. To investigate this effect further, we show both the salinity and tracer concentration on the release isopycnal (Fig. 3j-1). These two variables are also plotted against one another as a scatter plot (Fig. 3m-o). Two to three years into the experiment (i.e. during the late 2010 and 2011 voyages), higher tracer concentrations are found where the highest salinities are measured on the isopycnal. The core of the tracer is apparently moving with or close to the salinity maximum on the isopycnal.

On an isopycnal surface, the isopycnal salinity anomaly $\left(S^{\prime}\right)$ is defined as the local salinity minus some reference salinity $\left(S_{0}\right)$. We define $S_{0}$ on each density layer to be the salinity at the cast where the largest tracer concentration 

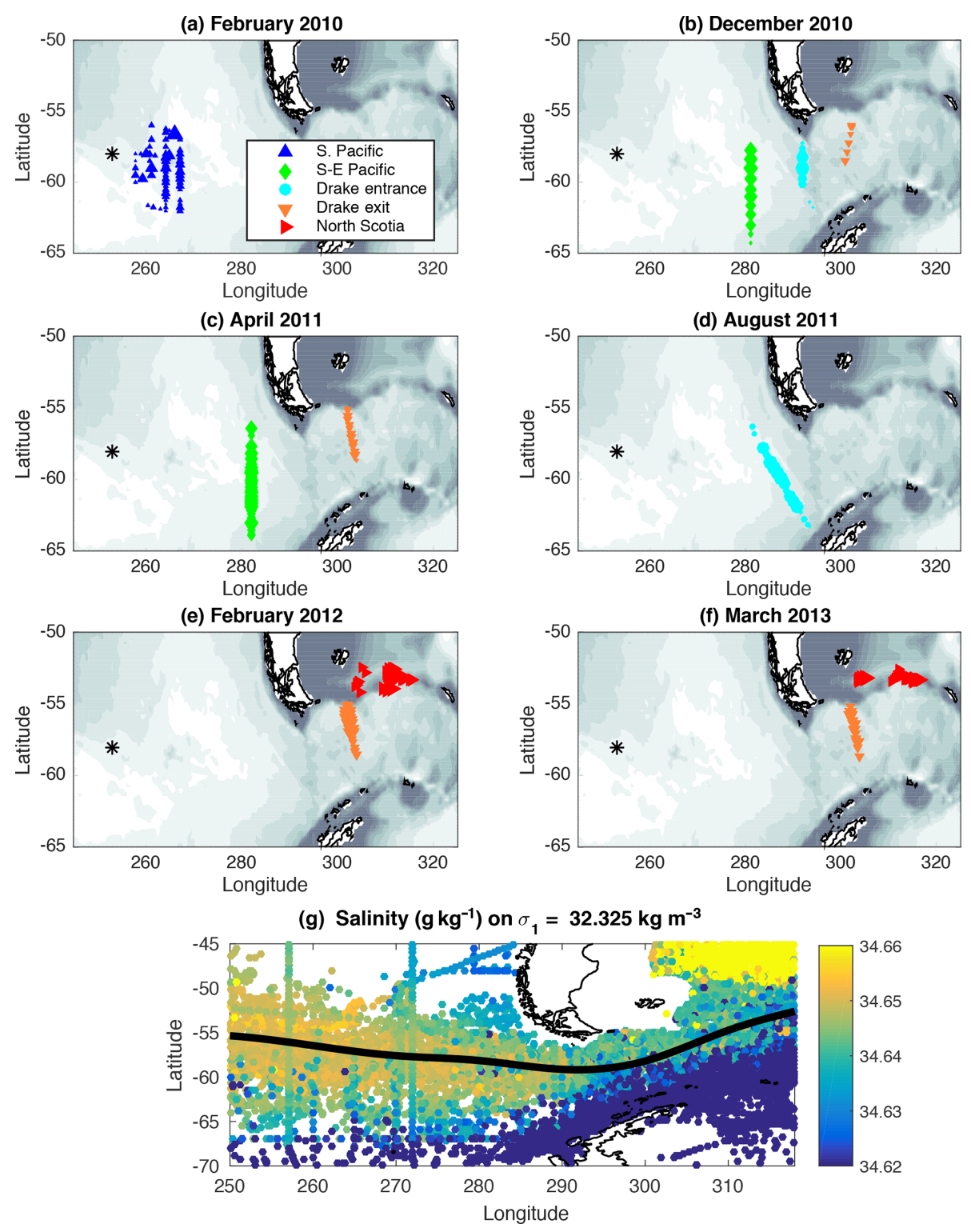

Figure 1. (a-f) Cast locations for each summer voyage in which tracer measurements were taken. The size of each marker corresponds to the peak tracer concentration for that cast. Marker sizes are scaled relative to the largest measured tracer concentration for that time period (i.e. markers corresponding to the cast where the largest concentration was measured have the same size between all six figures; see Fig. 2 for maximum concentrations for each section). The star indicates the release location, upward triangles the South Pacific region (US Voyage 2), diamonds the south-east Pacific section (along $282^{\circ}$ E), circles the Drake Entry section (along Phoenix Ridge), downward triangles the Drake exit section (otherwise known as SR1b) and right triangles the North Scotia section. (g) Salinity observations interpolated onto the release isopycnal surface (circles). A spline fit of the mean latitude of salinities between 34.645 and $34.65 \mathrm{~g} \mathrm{~kg}^{-1}$ is shown with a black line.

is measured. In order for the tracer to disperse in this coordinate, it must either cross isohalines at constant density (i.e. changing salinity with a compensating change in temperature) or cross isopycnal surfaces. Our focus here is on dispersion in the isopycnal direction.

We define $S_{0}$ separately for each section, rather than as the salinity value on which the tracer was injected. This is in part because as denser and lighter isopycnals are reached by the tracer, their reference salinity may change, and in part to account for migration of the peak due to gradual along-isopycnal transport. On the release isopycnal $S_{0}$ remains close to $34.65 \mathrm{~g} \mathrm{~kg}^{-1}$ throughout our study domain. $S_{0}$ appears to decrease slightly toward the east, but the trend is not robust and $S_{0}$ is always larger than $34.645 \mathrm{~g} \mathrm{~kg}^{-1}$.

In Fig. 4, the tracer distributions for each section are mapped onto density-versus-salinity-anomaly coordinates. While in geographical coordinates the dispersion of the tracer has multiple maxima and minima and appears to contract 

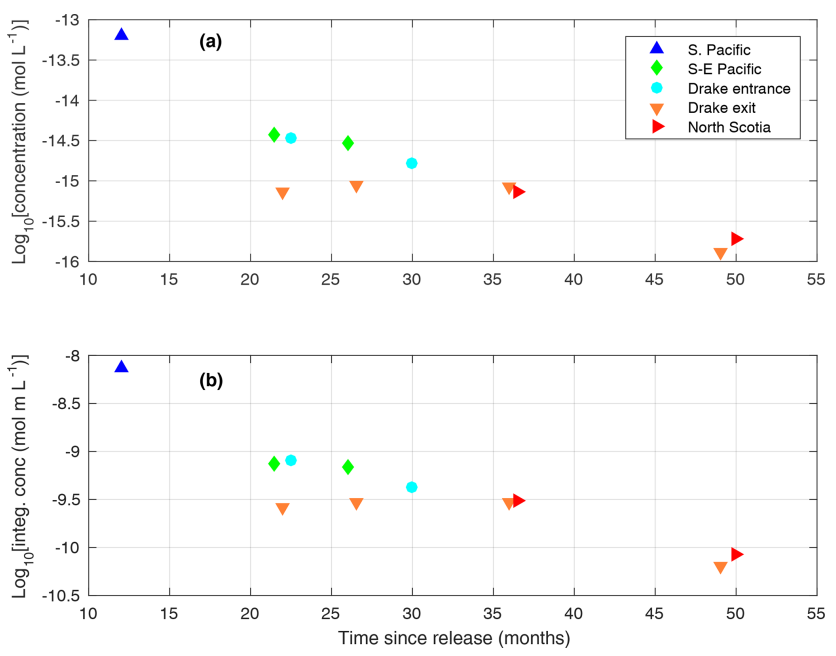

Figure 2. (a) The peak concentration measured $\left(\log _{10} \mathrm{molL}^{-1}\right)$ at each section as a function of time since release (months). (b) Peak depth-integrated concentration $\left(\log _{10} \mathrm{~mol} \mathrm{~mL}^{-1}\right)$. Although higher concentrations could be inhomogeneously distributed either side of the sections at a given time, the time series suggests that the concentration peak is closer to the eastern South Pacific before 30 months and closer to or beyond the North Scotia Ridge by the last transect near 50 months.

meridionally with time, in density-versus-salinity-anomaly coordinates the tracer spreads out more monotonically.

In order to quantify the spreading of the tracer in isopycnal salinity anomaly coordinates, we apply a non-linear leastsquares fit (using Matlab's fminsearch algorithm) of a twodimensional Gaussian distribution in that reference frame. This is done at each section to yield the standard deviation of salinity in the along-isopycnal direction. As mentioned above, growth of the tracer patch in the diapycnal direction during the initial 2 years of the experiment was analysed by Ledwell et al. (2011) and by Watson et al. (2013) and further discussion of diapycnal mixing is left to future work.

The evolution of the second moment of the tracer in isopycnal salinity anomaly coordinates (the square of the standard deviation) is shown in Fig. 5. There is an apparent weak spreading of the tracer in the first 24 months, with the tracer patch growing to second-moment values generally less than $5 \times 10^{-6}\left(\mathrm{~g} \mathrm{~kg}^{-1}\right)^{2}$; then, over the subsequent 24 months, more rapid spreading of the tracer occurs, reaching secondmoment values of the order of $5 \times 10^{-5}\left(\mathrm{~g} \mathrm{~kg}^{-1}\right)^{2}$.

To account for the various sources of sampling error, we estimate uncertainty using the following bootstrapping method. For each section, the tracer observations are randomly subsampled allowing for repeated sampling of the same data. For a section with $N$ samples, $N$ observations are chosen at random (with repetition), and the second moment is determined. This is then repeated $N$ times. Each estimate is shown in Fig. 5.
The uncertainty analysis reveals a larger range of secondmoment estimates for the initial South Pacific survey after 12 months than in surveys conducted after 20-30 months in the same region, potentially indicating an initial lack of correspondence between salinity and tracer filaments (Fig. 5). Uncertainties then increase for the later period and in the Scotia Sea, likely due to reduced tracer concentrations, poor coverage of the Gaussian distribution and a lack of consistency of the Gaussian model due to anisotropic variations in mixing and a changing background salinity and density field.

Nonetheless, there is a notable increase in the rate at which the tracer spreads in isopycnal salinity anomaly coordinates between the initial 2 years and the subsequent years of the experiment. This could be explained by the natural phases of evolution of the tracer patch, a change in the rate at which the tracer is mixed or by a change in background salinity gradient. In the next section, we investigate these explanations.

\section{Projection from isopycnal salinity anomaly to distance coordinates}

In order to interpret our analysis in terms of geographical dispersion, we next relate the isopycnal salinity anomaly coordinate to an equivalent cross-stream distance. We use the climatological mean distance between isohalines along the ACC to effect this transformation. Although such a transformation results in information loss relating to the moving salinity coordinate, it is instructive in linking dispersion to an apparent mixing coefficient for comparison with other work.

Distances between isohalines are determined using the entirety of available hydrographic data for the region. The hydrographic compilation used in this study includes ship-based observations from the World Ocean Database (http://www.nodc.noaa.gov/OC5/SELECT/ dbsearch/dbsearch.html, last access: 9 March 2020) and from the Argo programme (http://www.argo.ucsd.edu, http://argo.jcommops.org, last access: 9 March 2020), as well as from ship-based observations directly obtained from individual principal investigators (PIs) (see Sallée et al., 2010). Ideally, distances between isohalines would be determined for the precise period of the DIMES experiment, but due to data sparsity we have used the entire database.

For each vertical cast, potential density and salinity are determined. Salinity is then linearly interpolated onto the $\sigma_{1}=$ $32.325 \mathrm{~kg} \mathrm{~m}^{-3}$ isopycnal surface (Fig. 1f). Maps of these raw salinity values show a clear ridge of high salinity running along the ACC on this isopycnal. Higher salinity gradients are apparent further to the east as the ACC contracts. It is along this ridge that the tracer is released.

If fine-resolution (e.g. eddy-resolving), real-time and realistic salinity data were available on the isopycnal surface, we would form a time-varying salinity coordinate by calculating the area contained between salinity ranges and remap this onto a quasi-meridional distance (e.g. following Mar- 

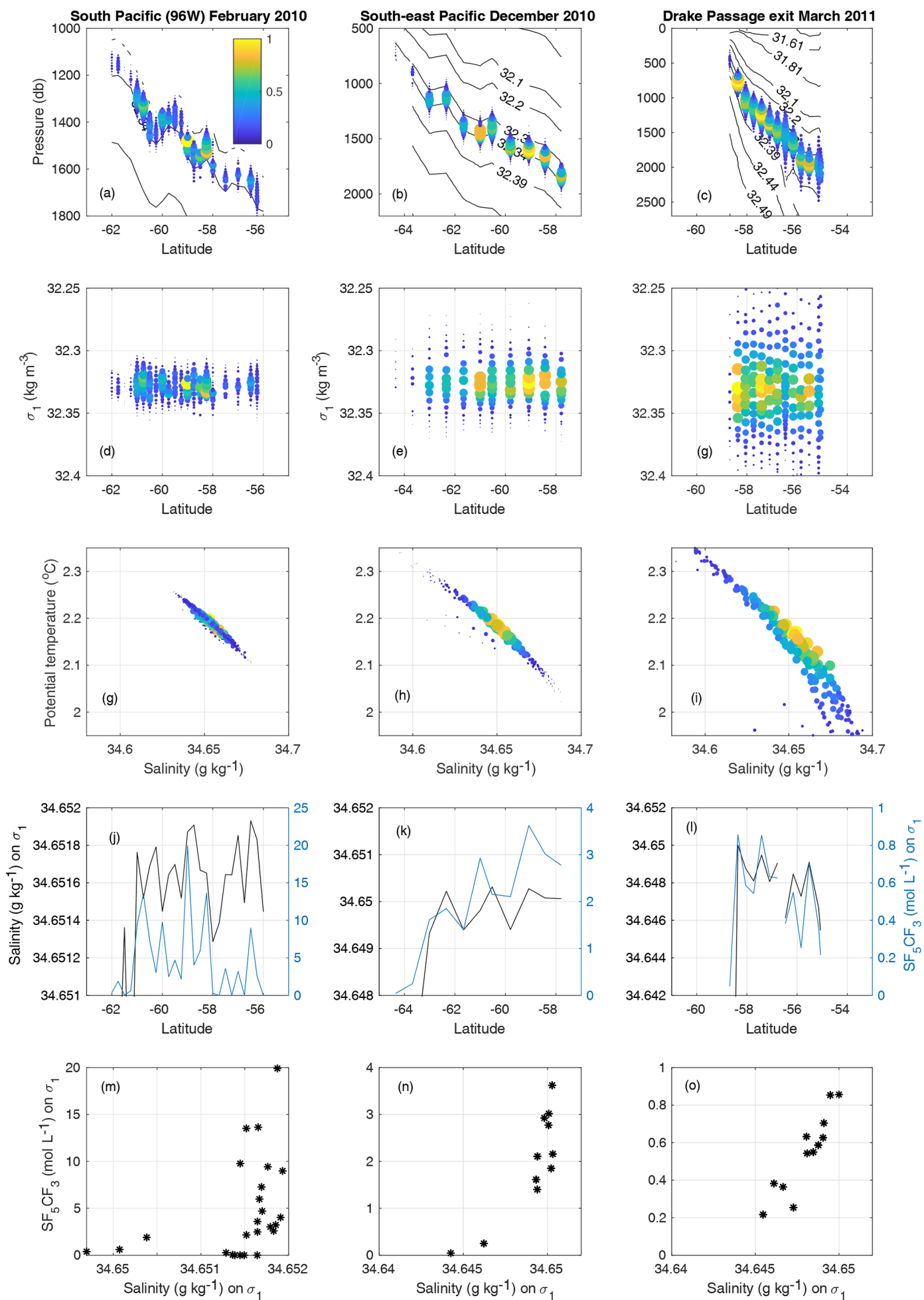

Figure 3. (a-c) Depth and latitude of tracer measurements, with contours of potential density referenced to $1000 \mathrm{~m}\left(\sigma_{0}\right.$; not evenly spaced). The Polar Front and the Subantarctic Front are where the slope of the density surfaces is large, near the southern and northern ends of these sections. (d-f) Potential density and latitude of tracer measurements. (g-i) Potential temperature and salinity. (j-l) Salinity and tracer on the $\sigma_{1}=32.325 \mathrm{~kg} \mathrm{~m}^{-3}$ surface. (m-o) Scatter plot of salinity and tracer concentration on the $\sigma_{1}=32.325 \mathrm{~kg} \mathrm{~m}^{-3}$ surface (note the changing $y$ axis range). For panels (a)-(i), colour represents tracer concentration relative to the peak measured for that section. Panels (a), (d) and (g) are from the South Pacific cruise of February 2010; (b), (e) and (h) the south-east Pacific section of December 2010; (c), (f) and (i) the Drake exit section of March 2011. 

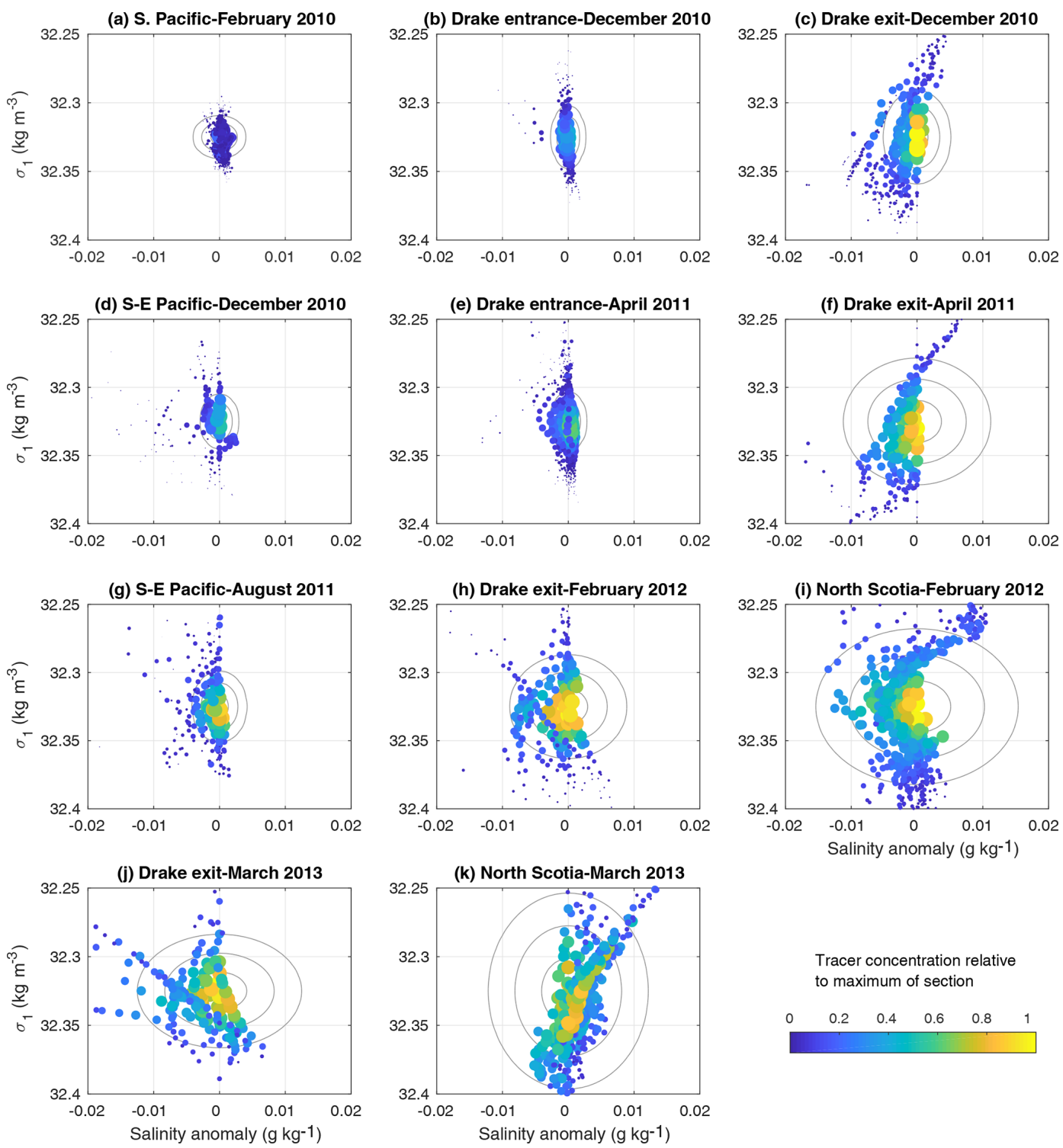

Figure 4. Data from all 11 sections from the five DIMES cruises discussed are shown in potential density-versus-isopycnal-salinity-anomaly coordinates. Each point is coloured by the tracer concentration relative to the maximum of that section (see Fig. 2 for these values). Grey contours show the $\sigma / 2, \sigma$ and $3 \sigma / 2$ contours of a fit of a 2D Gaussian curve to these data.

shall et al., 2006). However, such a density of data is not available from an observational product. Instead, we estimate the distance coordinate directly from the in situ data. We do this by, first, estimating the mean location of the salinity contour along which the tracer is centred and, second, calculating the average distance from that contour to other salinity measurement sites.

The western South Pacific and South Atlantic are partitioned into seven bands between longitudes 250, 260, 270, $280,290,300,310$ and $320^{\circ} \mathrm{E}$. Within each band, we determine the average latitude of salinity observations that fall between 34.645 and $34.65 \mathrm{~g} \mathrm{~kg}^{-1}$ (using a simple arithmetic mean). This defines a latitude $\left(y_{0}(x)\right)$ for each of the seven bands and effectively determines the approximate path the tracer would follow if it did not mix irreversibly. We use $10^{\circ}$ and not narrower bands to avoid noise arising from a minimal number of salinity observations being made on the isopycnal. A smooth set of points every $0.5^{\circ}$ of longitude are then used to define the curve for $y_{0}(x)$, using a one-dimensional cubic spline interpolation.

For each isopycnal salinity anomaly observation $\left(S^{\prime}\right)$ we determine its distance $\left(D\left(S^{\prime}\right)\right)$ from the curve $y_{0}(x)$. Distance is defined as the minimum great-circle distance from the observation to a point on the curve $y_{0}(x)$. The mean distance, $\overline{D\left(S^{\prime}\right)}$, is then the average of $D\left(S^{\prime}\right)$ for a given band of longitudes and over a range of salinities close to $S^{\prime} . \overline{D\left(S^{\prime}\right)}$ 


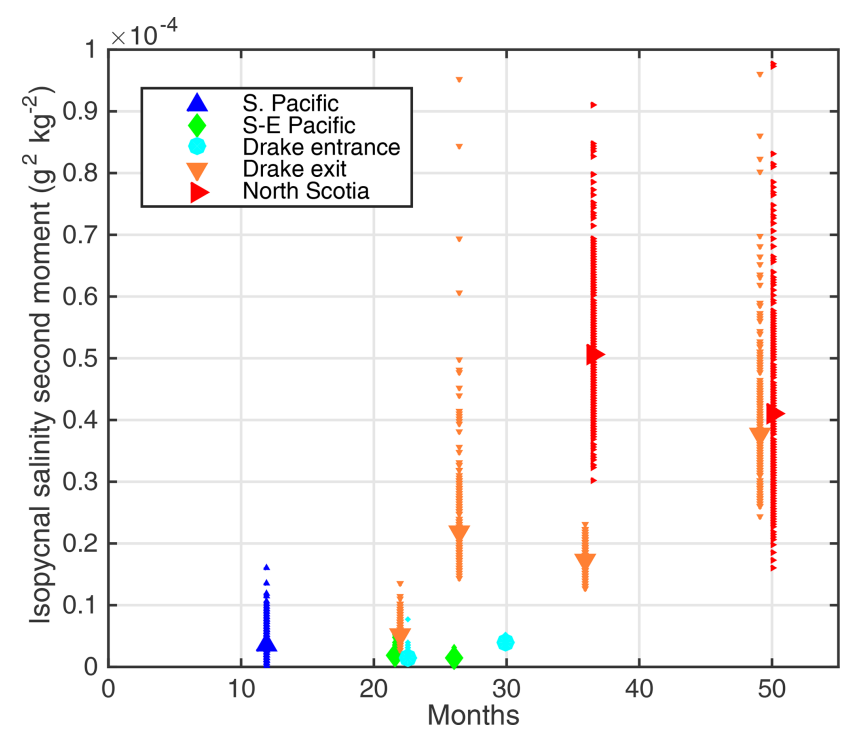

Figure 5. Isopycnal second moment of the tracer in salinity coordinates derived from the Gaussian fits in Fig. 3 versus time since release. Each small marker (making up the error bars) represents a single member of the bootstrap ensemble, and the larger marker the result of using all the data.
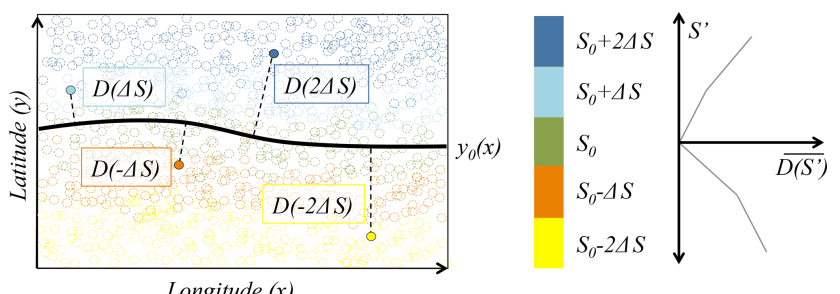

Figure 6. Schematic describing the method by which the mean distance as a function of salinity anomaly $\left(\overline{D\left(S^{\prime}\right)}\right)$ is calculated. Circles represent salinity observations on an isopycnal surface. The latitude of observations close to the target salinity $\left(S_{0} \pm \Delta S / 2\right.$; green) are used to define a locus of latitudes $\left(y_{0}(x)\right.$; solid line). The minimum great-circle distance $(D)$ of each observation from $y_{0}(x)$ is then determined and binned as a function of the salinity anomaly $\left(S^{\prime}=S-S_{0}\right)$. Distances are averaged within each bin, giving a relationship between salinity anomaly and distance $\overline{D\left(S^{\prime}\right)}$.

is used to transform the salinity anomaly coordinate into an equivalent distance coordinate. In regions where $y_{0}(x)$ follows a line of constant latitude (as is approximately the case in the South Pacific), $\overline{D\left(S^{\prime}\right)}$ is effectively the average meridional distance of water parcels with salinity $S^{\prime}$, from the line $y_{0}(x) . D\left(S^{\prime}\right)$ is averaged within each $10^{\circ}$ band and within salinity bins of $0.005 \mathrm{~g} \mathrm{~kg}^{-1}$, as shown in Fig. $7 \mathrm{~b}$.

Our distance coordinate is analogous to other pseudodistances used in diffusivity studies in the Southern Ocean, where quasi-stream-following coordinates are used. For example, Naveira Garabato et al. (2011) use a similar approximate conversion between dynamic height and latitudinal distance when estimating eddy stirring length scales. It should be noted that there is some unavoidable loss of spatial information in the use of water-mass-following coordinates. While the distance coordinate aims to relate changes in salinity at constant density to changes in distance, it is impossible to know the actual path taken by tracers, and so the distance conversion will always be approximate. We discuss the impact of changes in both the distance coordinate with longitude and their impact on apparent diffusivity in the next section.

The distance $\overline{D\left(S^{\prime}\right)}$ decreases substantially between the South Pacific and the Scotia Sea. At the release site in the South Pacific waters become $0.01 \mathrm{~g} \mathrm{~kg}^{-1}$ fresher over approximately $700 \mathrm{~km}$, while they change by the same amount over only $300 \mathrm{~km}$ in the Scotia Sea.

In order to translate the salinity second moment (Fig. 5) into units of distance squared (as relevant to the estimation of a mixing coefficient), we construct "reference profiles" based on $\overline{D\left(S^{\prime}\right)}$. The use of a reference profile is routine when converting isopycnally averaged tracer observations into vertical distance for the purpose of inferring a vertical diffusivity (Ledwell et al., 1993). Since there is a substantial change in the salinity gradient between the South Pacific and the Scotia Sea, and there is an apparent change in the rate at which the tracer spreads in salinity after 18-24 months, we have defined three mean profiles for the entire region between 250 and $310^{\circ} \mathrm{E}$, a western region between 250 and $290^{\circ} \mathrm{E}$, and an eastern region between 280 and $310^{\circ} \mathrm{E}$. The choice of longitudes here is arbitrary, and our aim is to merely assess the sensitivity of second moments and diffusivities estimated to a range of possible profiles.

Given distance as a function of salinity for each section, $\overline{D\left(S^{\prime}\right)}$, we use the climatological hydrography to map the tracer from isopycnal salinity-anomaly to distance-anomaly coordinates. The same distance-versus-salinity-anomaly profiles are used on all isopycnals.

We apply a non-linear least-squares fit of a twodimensional Gaussian distribution to each section to yield the standard width in the isopycnal direction $\left(\sigma_{\text {iso }}\right)$, with the resulting along-isopycnal component of the second moments shown in Fig. 8 now in the conventional units of squared metres.

\section{Interpretation of the isopycnal growth of the tracer patch}

Given the change in the isopycnal second moment at each section $\left(\Delta \sigma_{\text {iso }}^{2}\right)$ and the time between section occupations $(\Delta t)$, we can examine whether the tracer patch grows linearly, as would be expected from the ensemble area of a tracer patch with a constant diffusion coefficient $\left(K_{\text {iso }}\right)$, such that

$\Delta \sigma_{\text {iso }}^{2}=2 K_{\text {iso }} \Delta t$ 
(a) Average distance anomaly $\left(10^{5} \mathrm{~m}\right)$

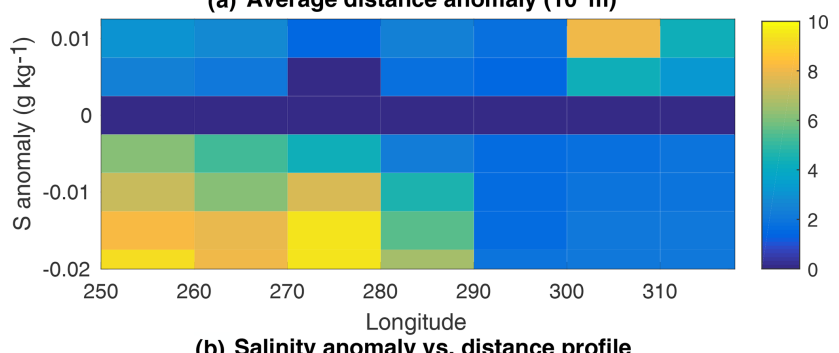

(b) Salinity anomaly vs. distance profile

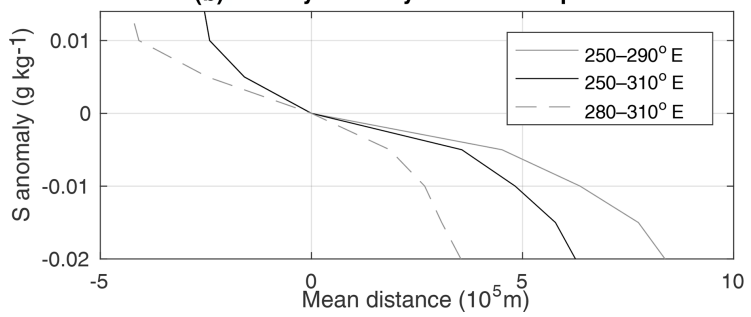

Figure 7. (a) The mean minimum distance of observations in each salinity class to the black line in $10^{\circ}$ longitude bands. (b) The mean salinity-versus-distance profile for the entire survey region and for a western and an eastern region. In the westernmost region there were insufficient high-salinity observations to produce a profile for the positive salinity anomalies there.

or if there is a temporal change in the growth rate either indicating different phases of the tracer evolution or geographical changes in $K_{\text {iso }}$.

In Fig. 8a, the second moments of the tracer are plotted as a function of time since release, using the mean salinityversus-distance profile for the entire survey region. The classical calculation of the eddy diffusion coefficient based on Lagrangian data statistics was first introduced by Taylor (1922), who showed that under stationary conditions and after multiples of the Lagrangian correlation time, the area of a cloud of particles will grow linearly in time at a rate known as the effective eddy diffusivity. In this paper, we describe the linear growth from an infinitesimally small second moment with an "apparent diffusivity". We next test this simple model of the growth of the tracer patch.

The apparent diffusivity is on the order of $50 \mathrm{~m}^{2} \mathrm{~s}^{-1}$ in the South Pacific leading up to the eastern South Pacific and Drake Entry sections and on the order of $400 \mathrm{~m}^{2} \mathrm{~s}^{-1}$ integrated over both the South Pacific and Scotia Sea regions. Downstream sections such as the Drake exit exhibit a large range of apparent diffusivities (i.e. when fitting secondmoment growth all the way back to the origin) from the order of $200 \mathrm{~m}^{2} \mathrm{~s}^{-1}$ to the order of $600 \mathrm{~m}^{2} \mathrm{~s}^{-1}$, with later diffusivities tending to be larger even though the tracer has transited through the same geographical domain.

The change in apparent diffusivity between the earlier period and the western region and the later period and the eastern region, can be explained by at least three mechanisms: first, the difference in salinity gradient from the eastern to western region could translate into a virtual increase in ap- parent diffusivity as we have not yet accounted for such variations; second, the tracer may enter a region of more vigorous isopycnal mixing in the east; third, the apparent diffusivity is low at the early stage after tracer release because the tracer is still growing slowly as it forms filaments, before achieving faster growth after some lag time on the order of months to years (Garrett, 1983) and coincidentally when it enters the eastern region. The later two possibilities are addressed in more detail in the discussion section below.

To ascertain whether the change in apparent diffusivity could be explained by a change in the salinity gradient itself, we estimate a diffusivity in two phases using salinity-versusdistance profiles for the western and eastern regions separately (shown in Fig. 7). For the western region, we use only tracer measurements made in the first 26 months of the experiment and to the west of $290^{\circ} \mathrm{E}$. These estimates assume that mixing is linear from the release date. For the eastern region, we use only measurements made after 20 months and to the east of $280^{\circ} \mathrm{E}$. The eastern region estimates are made by taking the difference in the second-moment estimates from the south-east Pacific and Drake Entry sections and those measured at least 6 months later at the Drake exit and North Scotia sections. Although the statistics of the diffusivity estimates are not normal, we estimate the error bounds based on the 16th and 84th deciles (equivalent to \pm 1 standard deviation for a normal distribution).

Considering only the sections in the western region in the first 26 months, this analysis yields a diffusivity of 40 $100 \mathrm{~m}^{2} \mathrm{~s}^{-1}\left(45-130 \mathrm{~m}^{2} \mathrm{~s}^{-1}\right.$ if the final Drake Entry section at 30 months is included). We estimate a diffusivity of 250 $870 \mathrm{~m}^{2} \mathrm{~s}^{-1}$ in the eastern region. This suggests that even when considering a change in the background salinity gradient there is still a substantial increase in diffusivity between the western and eastern region, so something else must explain this increase in diffusivity.

\section{Discussion}

In a previous study, data from DIMES together with output of a numerical model were used to quantify isopycnal mixing in the eastern South Pacific (Tulloch et al., 2014). Those authors estimated a diffusion coefficient using the ensemble area of the tracer patch and found it to be of the order of $700 \mathrm{~m}^{2} \mathrm{~s}^{-1}$. An additional study used float observations to estimate a diffusion coefficient on the order of $800 \mathrm{~m}^{2} \mathrm{~s}^{-1}$ (LaCasce et al., 2014). Here, we have taken a complementary approach and attempt to quantify the irreversible mixing of the tracer as it spreads both in the South Pacific and Scotia Sea regions. Using our novel coordinate framework and assuming linear growth from an infinitesimally small patch, we demonstrate that, as the tracer disperses, the evolution of the area of the tracer is consistent with an apparent diffusivity of $70 \pm 30 \mathrm{~m}^{2} \mathrm{~s}^{-1}$ in the south-east Pacific, increasing to $560 \pm 310 \mathrm{~m}^{2} \mathrm{~s}^{-1}$ downstream as the tracer enters the Scotia 
(a) $250-310^{\circ} \mathrm{E}$ distance vs. salinity profile

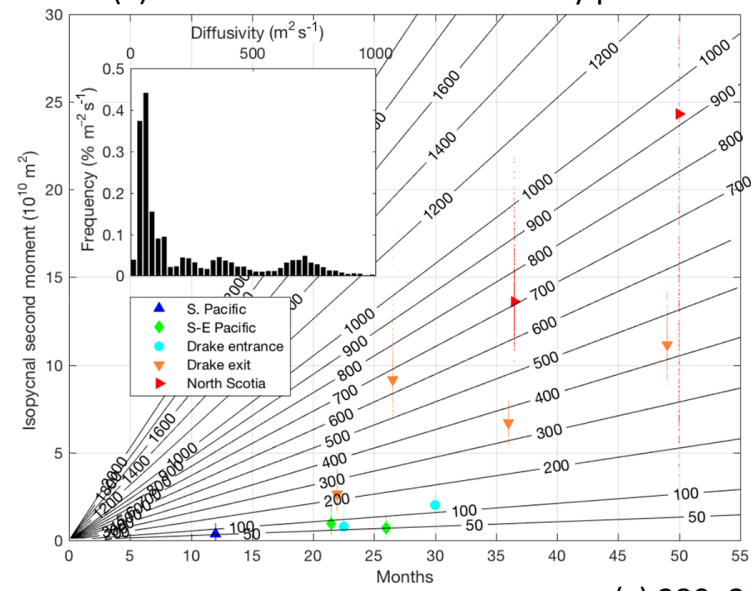

(b) $250-290^{\circ} \mathrm{E}$ profile

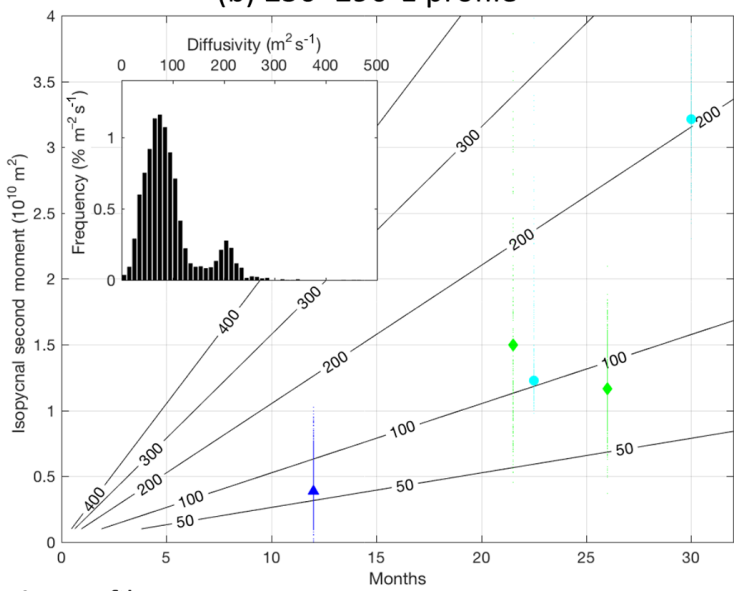

(c) $280-310^{\circ}$ E profile

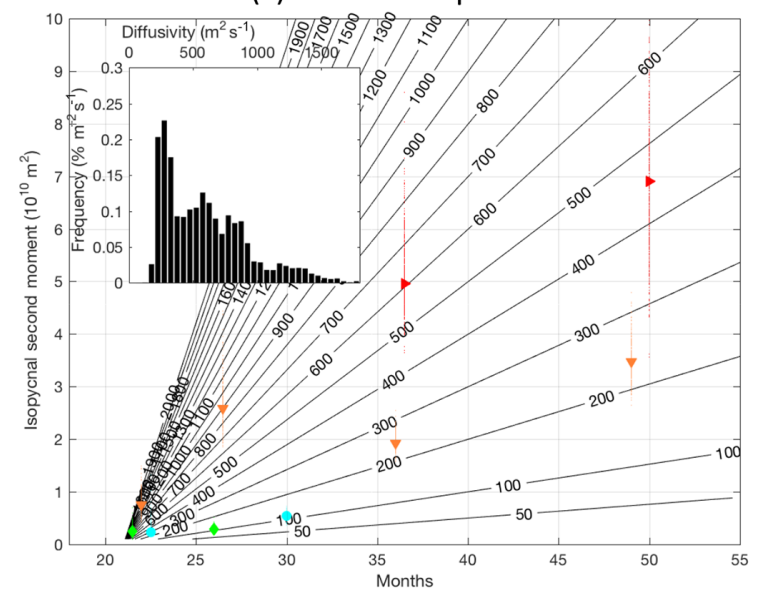

Figure 8. Isopycnal second moment of the tracer versus time since release, computed by projecting from tracer versus salinity anomaly into tracer versus distance, using a constant distance-versus-salinity profile. Panel (a) uses the profile from salinity data between 250 and $310^{\circ} \mathrm{E}$, (b) between 250 and $290^{\circ} \mathrm{E}$, and (c) 280 and $310^{\circ} \mathrm{E}$. Only tracer data gathered between those longitudes are shown. Each small marker represents a single member of the bootstrap ensemble and the larger marker the result of using all the data. The rate of change of the second moment in time is proportional to an apparent diffusivity (Eq. 1). Contours of apparent diffusivity are shown in black. Panels (a) and (b) show the case where the growth is linear from the release date, and (c) shows an offset of 21 months. Within each panel are shown the distribution functions of diffusivity estimates. In (a) and (b) these assume constant mixing from the release date. In (c) these estimates are based on the slope between the two eastern South Pacific sections and the two Scotia Sea sections.

Sea. While our estimate of apparent diffusivity in the Scotia Sea is consistent with Tulloch et al. (2014) and LaCasce et al. (2014), we note that our estimate in the south-east Pacific is significantly lower than their estimate.

While we cannot here disentangle the exact reason for such a difference with previous authors for our estimate in the south-east Pacific, we explore whether the increase in dispersion from the south-east Pacific to the Scotia Sea could be explained by the time evolution of the regime of dispersion of the tracer patch rather than by a regional difference in conditions. In other words, we explore the possibility that the tracer dispersion is initially very slow and rapidly increases after some time lag, making a linear growth assumption like we did above inappropriate. Indeed, Garrett (1983) discussed that the evolution of a tracer patch (that has already formed an approximately Gaussian distribution; the DIMES tracer was initially dispersed in a $20 \mathrm{~km}$ by $20 \mathrm{~km}$ cross shape) should have two distinct phases in its evolution: one first phase where the tracer forms streaks, so where the actual area of the tracer grows slowly; and a second phase after some time lag as the actual area approaches the area of an ellipse surrounding the tracer; the growth rate of the actual area of the tracer then asymptotes toward linear growth.

The time lag and time evolution of the area of the tracer patch in the two different regimes can be expressed as a function of a number of parameters of the flow field (Garrett, 1983, see the Appendix). By trying to best estimate those parameters and bootstrapping a best fit to our observed tracer patch dispersion with the Garrett (1983) theoretical prediction, we are able to estimate the isopycnal mixing $K_{\text {iso }}$ 
reached in the phase of linear growth of the tracer patch, as well as the time lag at which this regime is reached (see the Appendix). From the ensemble of solutions, we find that the isopycnal diffusivity lies between 240 and $550 \mathrm{~m}^{2} \mathrm{~s}^{-1}$ (Fig. Ala shows the distribution). The lag times are between 20 and 32 months. If we use the much lower small-scale diffusivity $\left(K_{\mathrm{S}}=0.04 \mathrm{~m}^{2} \mathrm{~s}^{-1}\right.$; see Appendix), the solutions for $K_{\text {iso }}$ and the lag time do not change substantially ( $K_{\text {iso }}=$ $290-600 \mathrm{~m}^{2} \mathrm{~s}^{-1}$; lag time $=19-29$ months). Both the results of Tulloch et al. (2014) and LaCasce et al. (2014) are consistent with the linear growth rate that we find here of 240 $550 \mathrm{~m}^{2} \mathrm{~s}^{-1}$. This linear growth sets in after an initial slow growth phase, as predicted by the theory of Garrett (1983) and suggested by our observation of a step change in diffusivities between the South Pacific and Scotia Sea. Additionally the lag time is consistent with the point at which we note the change in diffusivities.

Above we are not arguing that there is necessarily a large increase in effective isopycnal stirring between the Pacific and Atlantic in the Southern Ocean. We are pointing out that an increase in the rate of irreversible mixing of a tracer patch, as our analysis suggests, is expected (Garrett, 1983) even without changes in rates of eddy stirring or small-scale mixing.

Alternative explanations for the spread of apparent isopycnal diffusivities in Fig. 8 cannot be ruled out. It is possible that frontal suppression inhibited mixing in the South Pacific and that this broke down as the tracer entered Drake Passage, although this interpretation would arguably be at odds with the diffusivity estimates made by Tulloch et al. (2014) and LaCasce et al. (2014). Alternatively, the tracer growth may not have reached the lag time and may still be in a relatively weak mixing regime (potentially explaining why the implied diffusivities are on the low side of Tulloch et al. (2014) and LaCasce et al. (2014)). In this case, it may be that geographical changes in vertical mixing drive geographical changes in the size of filaments and therefore the apparent isopycnal mixing inferred (Smith and Ferrari, 2009). Vertical mixing was indeed found to increase as the tracer moved from the Pacific Ocean into the Scotia Sea (Watson et al., 2013).

We have neglected the effects of along-stream mixing in this analysis as is customary in vertical mixing calculations (Ledwell et al., 1993). In principle, if the tracer is mixed at the same rate in the along-stream direction at all salinities, the maximum concentration of the tracer will reduce but the second moment will be preserved. If, however, the tracer is mixed at different rates at different salinities (for example, it is mixed strongly close to the release salinity and weakly further away), then this could distort the second moment and therefore affect our diffusivity estimates.

\section{Conclusions}

Observations of a passive tracer released in the South Pacific Ocean have been discussed and we have projected tracer observations onto isopycnal salinity anomaly coordinates. The proposed coordinate is equivalent to isopycnal temperature anomaly and is stream-following. For the tracer to deviate from a line of constant temperature and salinity, irreversible transformations must occur. Spreading of the tracer in this coordinate hence relates to irreversible mixing.

The second moment of the tracer in salinity coordinates grows very slowly initially to the order of $0.05 \mathrm{~g}^{2} \mathrm{~kg}^{-2}$ in the South Pacific over the first 20-25 months. The initially tight correlation between salinity at constant density and the tracer could be exploited in future tracer release campaigns to predict where the core of the tracer might be found. Essentially if one is looking for a tracer in the deep ocean, the best place to look is likely to be at the same temperature and salinity (and therefore density) that the tracer was released at.

The second moment then grows to the order of $0.4 \mathrm{~g}^{2} \mathrm{~kg}^{-2}$ by 50 months, suggesting a change in the rate at which the tracer spreads through isopycnal salinity space. In order to relate dispersion in salinity coordinates to isopycnal mixing in geographical coordinates, we have related isopycnal salinity to cross-stream distance in the same way that density has been related to depth in previous studies. We have estimated the growth of the second moment of the tracer in these equivalent geographical coordinates.

In distance coordinates, the isopycnal second moment grows slowly in the first 26 months at the order of $75 \mathrm{~m}^{2} \mathrm{~s}^{-1}$, then rapidly thereafter at the order of $550 \mathrm{~m}^{2} \mathrm{~s}^{-1}$. The initial slow growth of the area of a tracer patch proposed by Garrett (1983) is able to explain these two regimes, although does not entirely preclude alternative explanations. Based on these data, the predicted lag time before the onset of linear growth of the tracer patch area is $20-32$ months. The rate of isopycnal mixing (representative of the large-scale dispersion of the tracer) is predicted to be $240-550 \mathrm{~m}^{2} \mathrm{~s}^{-1}$ and is consistent with, although somewhat lower than, two recent studies of the same region and period. 


\section{Appendix A}

According to Garrett (1983), a tracer patch that has already formed an approximately Gaussian distribution (the DIMES tracer was initially dispersed in a $20 \mathrm{~km}$ by $20 \mathrm{~km}$ cross shape) should have two distinct phases in its evolution:

1. The tracer forms streaks. The area of an ellipse surrounding the tracer grows much faster than the actual area of the tracer $(A)$, which evolves according to

$A=\pi \frac{K_{\mathrm{s}}}{\gamma} e^{\alpha \gamma\left(t-\frac{1}{4} \gamma^{-1}\right)}$,

where $K_{\mathrm{S}}$ is a small-scale diffusivity, $\gamma=\sqrt{u_{x}^{2}+v_{y}^{2}}$ is the strain rate $\left(u_{x}\right.$ is the zonal gradient of the zonal velocity, and $v_{y}$ is the meridional gradient of the meridional velocity), and $\alpha$ is a constant of the order of 1 .

2. As the actual area approaches the area of an ellipse surrounding the tracer, the growth rate of the actual area of the tracer then asymptotes toward linear growth, where $K_{\text {iso }}$ obeys (Eq. 1). We term the time at which Eqs. (1) and (A1) give the same value for $A$ and $\sigma_{\text {iso }}$ the lag time (specifically referred to as $t_{2}$ in Garrett (1983)).

Using typical values for the stratification and strain in the ocean, Garrett (1983) estimated the lag time to be on the order of 1 year. To complete this calculation for the region in question, the small-scale diffusivity $\left(K_{\mathrm{s}}\right)$, strain $(\gamma)$ and $\alpha$ are required. We use the small-scale diffusivity estimated by Boland et al. (2015) of $20 \mathrm{~m}^{2} \mathrm{~s}^{-1}$. As noted by Boland et al. (2015), this value is about 3 orders of magnitude larger than computed from the equation proposed by Young et al. (1982) and used in the Garrett (1983) study. It is also 1 order of magnitude larger than the $2-3 \mathrm{~m}^{2} \mathrm{~s}^{-1}$ estimated by (Ledwell et al., 1993) for a tracer patch at $300 \mathrm{~m}$ depth in the eastern North Atlantic. We therefore test the sensitivity of the lag time to this choice below. To determine the strain $(\gamma)$, we use SatGEM data (Meijers et al., 2011) mapped onto the release isopycnal and interpolated onto the $y(\bar{S})$ line defined in Sect. 4 based on satellite and hydrographic data from the DIMES survey period. Between 250 and $290^{\circ} \mathrm{E}$, we find $\gamma \approx 2 \times 10^{-6} \mathrm{~s}^{-1}$.

The most significant uncertainty in the estimation of the lag time lies in our choice of $\alpha$. As most of the variation from site to site of the dispersion process is presumably captured by $\gamma$, one might expect $\alpha$ to be roughly the same at each site of mesoscale stirring. The variation in $\alpha$ would quantify changes in the statistics of the strain other than its variance, for example variations in the Lagrangian autocorrelation of the strain rate.

Given the above numbers and using $\alpha=0.5$ (the value adopted by Garrett, 1983) we obtain a lag time of 2 months. With $\alpha=0.2$ (the value estimated from a simulated tracer release in the North Atlantic by Lee et al., 2009), the lag

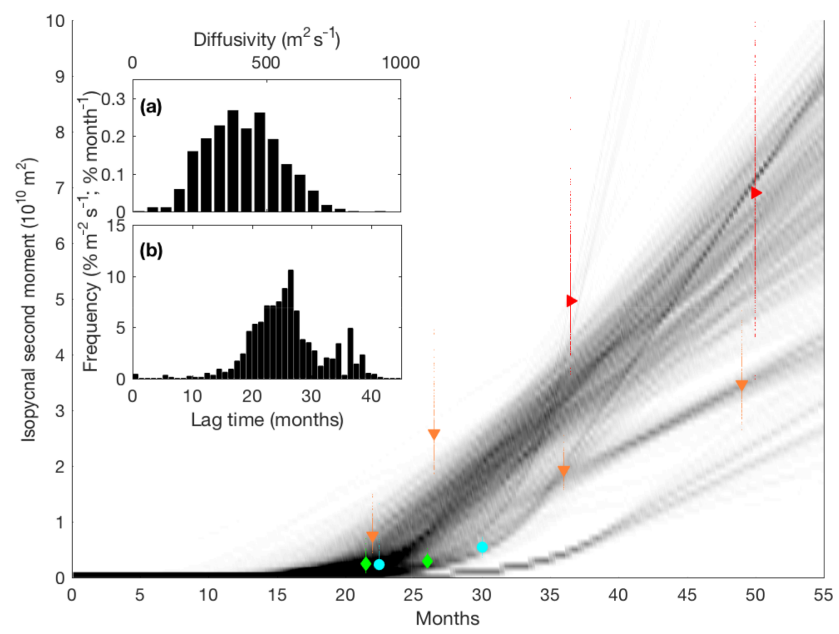

Figure A1. As in Fig. 8, but with the shading showing the density of solutions from 1000 best fits of the Garrett (1983) model where the sections are randomly subsampled. The two subplots show the distribution of isopycnal mixing coefficients (a) and lag times (b).

time increases to 6 months, and for $\alpha=0.1$ and 0.05 the lag times are 13 and 29 months, respectively. We estimate the uncertainty in $\gamma$ to be $10 \%-20 \%$, by shifting the averaging window for the SatGEM fields by $10^{\circ}$ to the east and west. Although the strain rate could be diagnosed in a range of ways, potentially leading to a larger uncertainty, it is likely that $\gamma$ has less influence on the uncertainty in Eq. (A1) than $\alpha$.

As the lag time is uncertain, we choose to use Eq. (1) to solve for both $K_{\text {iso }}$ and $\alpha$ using our estimates of $\sigma_{\text {iso }}^{2}$. In order to capture the behaviour of slow followed by fast growth phases, we match Eq. (1) to Eq. (A1) for the slow growth period with $\sigma_{\text {iso }}^{2}=A / 2 \pi$. Implicit in this fitting procedure is the assumption that, during the initial growth phase, our second moments, based on the tracer projected from salinity to distance coordinates, are related to the total area of the tracer patch. We cannot support this with theory. We simply use Eq. (A1) as a model that offers the behaviour of slow followed by fast growth. We only interpret the inferred lag time and $K_{\text {iso }}$ that describe the linear growth phase.

Using the entire ensemble of $\sigma_{\text {iso }}^{2}$ estimates, we solve for $K_{\text {iso }}$ and $\alpha$ such that the continuous line matching Eqs. (A1) and (1) at the lag time gives the best fit to the estimated second moments (using Matlab's fminsearch algorithm). We estimate the uncertainty again by bootstrapping, this time subsampling the 11 sections entering the estimate. That is, for each ensemble member, 11 non-unique random numbers are chosen and the corresponding section data are used to estimate $K_{\text {iso }}$ and $\alpha$. This process is repeated 1000 times to form an ensemble of $K_{\text {iso }}$ and $\alpha$ estimates. 
Data availability. The data are publicly available through the British Oceanographic Data Centre (http://www.bodc.ac.uk/ projects/data_management/international/dimes/, last access: 9 March 2020).

Author contributions. Author contributions, based on the CRediT model, were as follows: JDZ contributed to conceptualisation, formal analysis, investigation, methodology, validation, visualisation, writing as well as the original draft and writing along with review and editing; JBS contributed to conceptualisation, data curation, investigation, methodology, resources, software and writing as well as review and editing; AJSM contributed to conceptualisation, data curation, investigation, methodology, resources, software and writing as well as review and editing; ACNG contributed to funding acquisition, investigation, methodology, resources and writing as well as review and editing; AJW contributed to data curation, funding acquisition, investigation, methodology, resources, software and writing as well as review and editing; MJM contributed to data curation, funding acquisition, investigation, writing, and review and editing; BAK contributed to data curation, funding acquisition, methodology and software.

Competing interests. The authors declare that they have no conflict of interest.

Acknowledgements. The DIMES project was also supported by the National Science Foundation (NSF). We thank Jim Ledwell for many thoughtful contributions and discussions regarding this paper. We also thank all those who worked in the preparation and execution of the DIMES experiment. We thank the peer reviewers who provided helpful advice which led to improvements in this paper.

Financial support. This research has been supported by the Natural Environment Research Council (grant no. NE/E006000/1).

Review statement. This paper was edited by Ilker Fer and reviewed by two anonymous referees.

\section{References}

Boland, E. J. D., Shuckburgh, E., Haynes, P. H., Ledwell, J. R., Messias, M.-J., and Watson, A. J.: Determining a sub-mesoscale diffusivity using a roughness measure applied to a tracer release experiment in the Southern Ocean, J. Phys. Oceanogr., 45, 1610 1631,2015

Garrett, C.: On the initial streakness of a dispersing tracer in twoand three-dimensional turbulence, Dynam. Atmos. Oceans, 7, 265-277, 1983

Gnanadesikan, A., Pradal, M.-A., and Abernathey, R.: Isopycnal mixing by mesoscale eddies significantly impacts oceanic anthropogenic carbon uptake, Geophys. Res. Lett., 42, 4249-4255, 2015.
Gregory, J. M.: Vertical heat transports in the ocean and their effect on time-dependent climate change, Clim. Dynam., 16, 501-515, 2000.

Ho, D. T., Ledwell, J. R., and Smethie Jr., W. M.: Use of SF5CF3 for ocean tracer release experiments, Geophys. Res. Lett., 35, L04602, https://doi.org/10.1029/2007GL032799, 2008.

Iselin, C. O.: The influence of vertical and lateral turbulence on the characteristics of the waters at mid-depths, Eos Trans. AGU, 20, 414-417, https://doi.org/10.1029/TR020i003p00414, 1939.

Killworth, P.: An equivalent barotropic mode in the fine resolution AntArctic model, J. Phys. Oceanogr., 22, 1379-1387, 1992.

LaCasce, J., Ferrari, R., Marshall, J., Tulloch, R., Balwada, D., and Speer, K.: Float-derived isopycnal diffusivities in the DIMES experiment, J. Phys. Oceanogr., 44, 764-780, 2014.

Ledwell, J., Watson, A., and Law, C.: Evidence of slow mixing across the pycnocline from an open-ocean tracer experiment, $\mathrm{Na}$ ture, 364, 701-702, 1993.

Ledwell, J., Watson, A., and Law, C.: Mixing of a tracer in the pycnocline, J. Geophys. Res., 103, 21499-21529, 1998.

Ledwell, J. R., St. Laurent, L. C., Girton, J. B., and Toole, J. M.: Diapycnal mixing in the AntArctic circumpolar current, J. Phys. Oceanogr., 41, 241-246, 2011.

Lee, M.-M., Nurser, A. G., Coward, A. C., and De Cuevas, B. A.: Effective eddy diffusivities inferred from a point release tracer in an eddy-resolving ocean model, J. Phys. Oceanogr., 39, 894-914, 2009.

Marshall, J. and Speer, K.: Closure of the meridional overturning circulation through Southern Ocean upwelling, Nat. Geosci., 5, 171-180, 2012.

Marshall, J., Shuckburgh, E., Jones, H., and Hill, C.: Estimates and implications of surface eddy diffusivity in the Southern Ocean derived from tracer transport, J. Phys. Oceanogr., 36, 1806-1821, 2006.

Meijers, A., Bindoff, N., and Rintoul, S.: Estimating the fourdimensional structure of the Southern Ocean using satellite altimetry, J. Atmos. Ocean. Tech., 28, 548-568, 2011.

Morris, M. Y., Hall, M. M., St. Laurent, L. C., and Hogg, N. G.: Abyssal mixing in the Brazil basin, J. Phys. Oceanogr., 31, 33313348, 2001.

Naveira Garabato, A. C., Ferrari, R., and Polzin, K. L.: Eddy stirring in the Southern Ocean, J. Geophys. Res.-Oceans, 116, https://doi.org/10.1029/2010JC006818, 2011.

Naveira Garabato, A. C., Polzin, K. L., Ferrari, R., Zika, J. D., and Forryan, A.: A microscale view of mixing and overturning across the Antarctic Circumpolar Current, J. Phys. Oceanogr., 46, 233 254, 2016.

Rudnick, D. L. and Martin, J. P.: On the horizontal density ratio in the upper ocean, Dynam. Atmos. Oceans, 36, 3-21, 2002.

Sallée, J. B., Speer, K., Rintoul, S., and Wijffels, S.: Southern Ocean thermocline ventilation, J. Phys. Oceanogr., 40, 509-529, 2010.

Sallée, J.-B., Matear, R. J., Rintoul, S. R., and Lenton, A.: Localized subduction of anthropogenic carbon dioxide in the Southern Hemisphere oceans, Nat. Geosci., 5, 579-584, 2012.

Sijp, W. P., Bates, M., and England, M. H.: Can isopycnal mixing control the stability of the Thermohaline Circulation in ocean climate models?, J. Climate, 19, 5637-5651, 2006.

Smith, K. S. and Ferrari, R.: The production and dissipation of compensated thermohaline variance by mesoscale stirring, J. Phys Oceanogr., 39, 2477-2501, 2009. 
Sokolov, S. and Rintoul, S. R.: Circumpolar structure and distribution of the Antarctic Circumpolar Current fronts: 1. Mean circumpolar paths, J. Geophys. Res.-Oceans, 114, https://doi.org/10.1029/2008JC005108, 2009.

Sundermeyer, M. A. and Price, J. F.: Lateral mixing and the North Atlantic Tracer Release Experiment: Observations and numerical simulations of Lagrangian particles and a passive tracer, J. Geophys. Res.-Oceans, 103, 21481-21497, 1998.

Taylor, G. I.: Diffusion by continuous movements, P. Lond. Math. Soc., 2, 196-212, 1922.

Toole, J. M. and McDougall, T. J.: Mixing and stirring in the ocean interior. Ocean Circulation and Climate, International Geophysics Series, Academic Press, 77, 337-355, 2001.

Tulloch, R., Ferrari, R., Jahn, O., Klocker, A., LaCasce, J., Ledwell, J. R., Marshall, J., Messias, M.-J., Speer, K., and Watson, A.: Direct estimate of lateral eddy diffusivity upstream of Drake Passage, J. Phys. Oceanogr., 44, 2593-2616, 2014.

Veronis, G.: On properties of seawater defined by temperature, salinity, and pressure, J. Marine Res., 30, 227-255,, 1972.
Watson, A. J., Ledwell, J. R., Messias, M.-J., King, B. A., Mackay, N., Meredith, M. P., Mills, B., and Garabato, A. C. N.: Rapid cross-density ocean mixing at mid-depths in the Drake Passage measured by tracer release, Nature, 501, 408-411, 2013.

Young, W., Rhines, P., and Garrett, C.: Shear flow dispersion, internal waves and horizontal mixing in the ocean, J. Phys. Oceanogr., 12, 515-527, 1982.

Zika, J. D., McDougall, T. J., and Sloyan, B. M.: A tracer-contour inverse method for estimating ocean circulation and mixing, J. Phys. Oceanogr., 40, 26-47, 2009a.

Zika, J. D., Sloyan, B. M., and McDougall, T. J.: Diagnosing the Southern Ocean Overturning from tracer fields, J. Phys. Oceanogr., 39, 2926-2940, 2009 b.

Zika, J. D., Sommer, L., Dufour, J., J-M., C. M., B., B., Brasseur, P., Dussin, R., Penduff, T., D., I., Lenton, A., Madec, G., Mathiot, P., Orr, J., Shuckburgh, E., and Vivier, F.: Vertical eddy fluxes in the Southern Ocean, J. Phys. Oceanogr., 43, 941-955, 2013. 